Rodrigues, A., Cavallet, M., \& Galera, C. A. (2020). Memória de Trabalho: Uma nova fronteira para o

Revista Comportamento do Consumidor marketing. Consumer Behavior Review, 4(2), 162-175.

ISSN: 2526-7884

Editor: Prof. Dr. Marconi Freitas da Costa Journal's e-mail: cbr@ufpe.br
Evaluation: Double blind review

Received: 9 de fevereiro de 2020

Accepted: 28 de abril de 2020

\title{
MEMÓRIA DE TRABALHO: UMA NOVA FRONTEIRA PARA O MARKETING
}

\author{
Working Memory: A new framework for marketing
}

\author{
Airton Rodrigues ${ }^{1}$ \\ ORCID: https://orcid.org/0000-0002-5055-6352 \\ E-mail: airton.rodrigues@fatec.sp.gov.br \\ Mikael Cavallet ${ }^{2}$ \\ ORCID: https://orcid.org/0000-0002-2626-0121 \\ E-mail: mklcv@hotmail.com \\ Cesar Alexis Galera ${ }^{3}$ \\ ORCID: https://orcid.org/0000-0002-3021-7126 \\ E-mail: algalera@usp.br
}

${ }^{1}$ Faculdade de Tecnologia de São Paulo - Luigi Papaiz, Diadema, Brasil

${ }^{2}$ Centro Universitário do Vale do Ribeira, Registro, Brasil

${ }^{3}$ Programa de Pós-Graduação em Psicobiologia, Universidade de São Paulo, Ribeirão Preto, Brasil

\begin{abstract}
Resumo
A memória de trabalho recebeu exaustiva atenção da psicologia em diversos estudos experimentais que explicam o funcionamento da atenção e o processamento das informações visuais e fonológicas. Contudo, poucos deles direcionados ao comportamento do consumidor. 0 processo de decisão de compra acontece neste sistema cognitivo limitado na quantidade das informações que podem ser armazenadas e no tempo a serem retidas. Demonstramos o estado da arte de como este construto cognitivo é abordado nos estudos
\end{abstract}

\begin{abstract}
The working memory received exhaustive attention from psychology in several experimental studies that explain the functioning of attention and the processing of visual and phonological information. However, few of them are directed at consumer behavior. The purchase decision process takes place in this cognitive system limited in the amount of information that can be stored and the time to be retained. We demonstrate the state of the art of how this cognitive construct is addressed in studies on
\end{abstract}


sobre o comportamento do consumidor, envolvendo elementos de comunicação, precificação, varejo e decisão de compra. 0 artigo mostra a carência de produções em revistas indexadas no Brasil sobre este tema e apresenta oportunidades para os pesquisadores aprofundarem este conhecimento sob o olhar do comportamento do consumidor.

Palavras-chave: Memória de trabalho, Comportamento do consumidor, Memória de curto prazo, Neuromarketing. consumer behavior, involving elements of communication, pricing, retail, and purchase decision. The article shows the lack of productions in indexed magazines in Brazil on this topic and presents opportunities for researchers to deepen this knowledge from the perspective of consumer behavior.

Keywords: Working memory, Consumer behavior, Short term memory, Neuromarketing.

\section{INTRODUÇÃO}

Há mais de meio século, Willian Lazer e Eugene Kelley publicaram o artigo Interdisciplinary Horizons in Marketing (Lazer \& Kelley, 1960), naquele hoje que é um dos principais periódicos de Marketing, o Journal of Marketing. Neste trabalho seminal, os autores afirmam que o Marketing é uma disciplina em desenvolvimento e deve incorporar o conhecimento das outras disciplinas. Entre as diferentes interfaces do Marketing, um elo sólido e profundo se estabelece com a Psicologia. As duas disciplinas pertencem às Ciências Sociais, porém, enquanto a Psicologia pode ser compreendida como uma ciência pura, o Marketing é dependente de diferentes áreas do conhecimento (Barcelos \& Rossi, 2015).

Há uma evidente conexão entre ambas (talvez com o Marketing mais dependente da Psicologia), mas a relação de conhecimentos entre estas áreas não é sempre fluida nem bidirecional, e certamente depende de uma interface ativa de troca de informações. Publicações prestigiosas como Psychology and Marketing, Journal of Consumer Psychology, Journal of Marketing Behavior e Journal of Consumer Psychology, ajudam na tarefa de aproximar os dois campos do conhecimento. Apesar do rico conteúdo presente nestas publicações, ainda há certo descompasso teórico entre o Marketing e a Psicologia, sobretudo nas publicações nacionais. Especificamente, são escassos estudos sobre como os estímulos de marketing são processados pela memória de trabalho (ou memória operacional). E praticamente inexistentes quando se restringem à língua portuguesa. Esta é uma lacuna muito importante a ser preenchida, visto que este construto abriga processos relevantes do sistema de escolha de marcas e produtos.

A memória de trabalho e o processo de decisão de compra compartilham de estruturas cognitivas e funcionalmente operam sinergicamente no cérebro (Murray, Jaramillo, \& Wang, 2017). Portanto, estudar o comportamento cognitivo do consumidor e sua relação com preferências de marcas e produtos passa necessariamente pela compreensão de como os elementos de marketing se organizam na memória de trabalho. Aliás, é crescente o interesse de técnicas neurocientíficas para avaliar as respostas emocionais e cognitivas no comportamento de compra (Solnais, Andreu-Perez, Sánchez-Fernández, \& Andréu-Abela, 2013). 0 chamado neuromarketing consiste em um novo campo de estudos que procura obter informações sobre como funciona os processos mentais dos consumidores (Ratnayake, Broderick, \& Mitchell, 2010). Alguns dos pontos centrais de investigação da neurociência aplicada ao marketing são memória, cognição e atenção dos consumidores (Hammou, Galib, \& Melloul, 2013), processos onde a memória de trabalho possui um grande protagonismo.

Através de uma revisão narrativa, oferecemos o "estado da arte" dos estudos envolvendo a memória de trabalho no escopo do comportamento do consumidor e sugerimos oportunidades de estudos que possam levar a compreender melhor como as informações mercadológicas são processadas por este construto. Em um primeiro momento realizamos a conceituação da memória de 
trabalho, como está dividida e funcionalmente como opera as informações cognitivas. Segue, então, detalhes sobre o levantamento bibliográfico realizado e as evidências de como a memória de trabalho processa elementos de comunicação, preço, percepção de produtos e comportamento de compra. Variações na capacidade de processamento e/ou armazenamento da memória de trabalho podem ser responsáveis pela explicação das diferenças individuais no comportamento de compra (mais ou menos impulsivos) e na forma como produtos e propagandas são percebidos pelos consumidores.

Tanto a memória de curto prazo quanto a memória de trabalho são compreendidas como estruturas de conservação momentânea da informação: a diferença é que a memória de curto prazo realiza apenas o armazenamento temporário e passivo, enquanto que a memória de trabalho possui um processo ativo, tanto na retenção como na manipulação das informações que sustentam o processo de decisão (Aben, Stapert, \& Blokland, 2012). A memória de trabalho é um sistema de capacidade limitada (Radvansky \& Copeland, 2006) responsável pela ativação, gerenciamento e integração das informações provenientes do ambiente com outras informações retidas na memória de longo prazo, que propicia ao consumidor tomar decisões e regular o seu comportamento de compra (Alloway, Gerzina, \& Moulder, 2016; Rumpf et al., 2015).

Dentre os vários modelos que buscam descrever a estrutura da memória de trabalho, o mais aceito foi o modelo idealizado por Baddeley (Wingfield, 2016). Neste modelo, a memória de trabalho é composta por dois componentes modais (um armazenador visual e um armazenador verbal) e um armazenador não modal (o buffer episódico), ambos controlados por um mecanismo de atenção que assume o controle do sistema e direciona o foco de atenção, conforme a situação, associado a um componente hedônico (Baddeley, 1996; Baddeley, Banse, Huang, \& Page, 2012; Repovs \& Baddeley, 2006). A informação processada pelo executivo central pode ser direcionada para o laço fonológico (se for conteúdo verbal) ou para o esboço visuoespacial (se for visual) (Allen, Baddeley, \& Hitch, 2006; Baddeley, 1996, 2002). Apesar do armazenamento e processamento das informações do sistema serem realizadas por componentes especializados, a integração das informações é realizada por um buffer episódico, que cria uma representação única e complexa. Este buffer consolida as informações adquiridas a partir dos órgãos sensoriais, acrescida das informações armazenadas na memória de longo prazo e matizadas pelos aspectos emocionais provenientes da atuação do detector hedônico (Baddeley, 2013; Baddeley et al., 2012). É dessa forma complexa que as informações sensoriais se mantêm acessíveis na memória de trabalho e o produto deste processamento pode retroalimentar a memória de longo prazo com novas informações.

A memória de trabalho opera como uma via de mão dupla em relação à memória de longo prazo: ela envia e recebe informações a todo o momento, atuando como uma central de processamento on-line. A construção da decisão de compra e o comportamento do consumidor estão sujeitos a este mecanismo de processamento. As diferenças individuais no funcionamento da memória de trabalho, somadas às experiências presentes na memória de longo prazo, interferem na forma como os consumidores se relacionam com marcas e produtos (Lobo, Acrani, \& Ávila, 2008). É neste espaço cognitivo limitado que as informações são armazenadas temporariamente para construção do processo de decisão, contudo, este processo de decisão está sujeito à quantidade e relevância destas informações (Argyriou \& Melewar, 2011).

Existe uma relação direta entre a capacidade de armazenamento da memória de trabalho - que varia entre os indivíduos - e a quantidade de critérios que um consumidor poderá utilizar em uma decisão de compra (Bose et al., 2013; Rumpf et al., 2015). Aqueles que possuem a memória de trabalho com alta capacidade de armazenamento, conseguem operar uma quantidade maior de informações e podem utilizar um conjunto de regras de decisões mais elaboradas (Ester et al., 2014); além de estarem mais aptos a controlar comportamentos automáticos, isolando estímulos irrelevantes no processo de decisão (Corbin, McElroy, \& Black, 2010). Há relações entre a capacidade de armazenamento da memória de trabalho, a quantidade de critérios utilizados no processo de decisão de compra e o nível de impulsividade que um consumidor teria em adquirir um determinado produto. Indivíduos com menor capacidade na memória de trabalho poderiam ter o sistema de armazenamento sobrecarregado rapidamente, o que levaria à construção de regras mais simples para a decisão de compra, sendo o inverso também verdadeiro (Alloway et al., 2016). Esta redução na quantidade de critérios pode tornar os indivíduos menos rigorosos e mais suscetíveis a comportamentos impulsivos 
e, talvez, mais inconsequentes (Wilhelm; Tobias, Malte, Reinout \& Manfred, 2016). Disfunções na mediação entre os processos afetivos e a memória de trabalho poderiam levar a um comportamento de compra temerário ou até mesmo a aspectos patológicos do consumo.

A capacidade da memória de trabalho não se restringe apenas a armazenar e processar informações, mas também possui relações com o controle da atenção (Rumpf et al., 2015), o que certamente afeta a forma como a decisão é construída na mente do consumidor. Acredita-se que a atenção seja uma atividade de ordem superior que sustenta o processamento de informações realizado na memória de trabalho do consumidor, onde é mantida uma "cesta" limitada de produtos/marcas enquanto o consumidor elabora construções mentais para configurar sua escolha (Janiszewski, Kuo, \& Tavassoli, 2013). Caso novos estímulos capturem o foco de atenção, (eg.: propaganda sedutora de uma marca concorrente), as novas e as antigas informações podem competir pelos limitados recursos cognitivos e resultar na reordenação das informações disponíveis na memória de trabalho, com consequente perda de conteúdo, quer seja o antigo, previamente armazenado, ou o novo estímulo oferecido (Janiszewski et al. 2013).

A capacidade limitada da memória de trabalho levaria a uma quantidade fixa de produtos possíveis de serem armazenados neste espaço para a construção da escolha do consumidor, fazendo com que diferentes marcas se alternassem nesta "cesta de decisão" (Bleckley et al., 2015; Eriksson et al., 2015). E as diferenças individuais existentes na capacidade de armazenamento da memória de trabalho levaria a diferentes formas de configurar esta "cesta de decisão". Caso um determinado consumidor tenha uma maior capacidade de armazenamento de informações na memória de trabalho, ele estaria equipado com recursos para manipular uma quantidade maior de informações, sustentar um processo de decisão complexo e, ao mesmo tempo, gerenciar dinamicamente o foco de atenção de forma a considerar novas possibilidades de marcas, sem necessariamente descartar as marcas previamente armazenadas (Alloway et al., 2016). De forma inversa, pessoas com menor capacidade da memória de trabalho estariam mais suscetíveis à propaganda de impacto, pois poderiam desalojar marcas concorrentes da "cesta de decisão" mental do consumidor e reordenar o rol de possibilidades consideradas no processo de decisão.

Daniel Kahneman, observando a forma como respondemos às exigências apresentadas pelo ambiente, mostrou que as respostas individuais são mediadas por dois sistemas cognitivos diferentes (Kahneman, 2011). O Sistema 1, evolutivamente mais antigo, presente também em outros animais, é definido por um conjunto de subsistemas autônomos que reagem instintivamente e automaticamente às mudanças ambientais. Por exemplo, não precisamos pensar muito para fugir ao identificar a ameaça de um animal peçonhento. 0 Sistema 1 responde a essas situações de maneira automática (Evans, 2003; Kahneman, 2011). O Sistema 2 é evolutivamente mais recente e, pode-se supor, eminentemente humano. Este sistema é responsável pela seleção de respostas para situações nas quais o Sistema 1 não é eficiente. Nas condições que exigem respostas novas, para as quais não podemos reagir instintivamente ou, pelo contrário, quando devemos inibir respostas impulsivas, entra em cena o Sistema 2, responsável pelo raciocínio abstrato e hipotético, que está, de maneira geral, correlacionado com medidas de inteligência geral (Evans, 2003). A memória de trabalho, elemento central do Sistema 2 (Evans, 2003), afeta diretamente o ritmo dos processos cognitivos: tanto em termos da quantidade como da frequência de conceitos ativados para construção do processo de decisão (Stocchi, Wright, \& Driesener, 2003).

0 processo de escolha do consumidor pode acontecer de duas formas: mediante um processo intuitivo e automático (muito orientado pelo Sistema 1) ou de forma deliberativa, fruto de um processo de reflexão (fundamentado no Sistema 2). Uma vez que a memória de trabalho é um elemento central no Sistema 2, percebe-se claramente que a limitação no armazenamento pode levar ao esgotamento dos recursos cognitivos, prejudicando a tomada de decisão deliberada e favorecendo a decisão intuitiva (Dhar \& Gorlin, 2013). Enquanto o Sistema 2 não estiver sobrecarregado, poderá funcionar como um "freio" às ações impulsivas, operando como um mediador racional do comportamento (Hofmann et al., 2008). Isto explica um pouco sobre o comportamento impulsivo de compras. Por outro lado, mesmo que a decisão seja orientada pelo Sistema 1, a atuação da memória de trabalho, através de processos deliberativos acerca da decisão a ser tomada, pode infringir alguns limites a este comportamento impulsivo (Dhar \& Gorlin, 2013). 
Observadas as características do Sistema 1 e do Sistema 2, é possível dizer que as decisões de compra estão fundamentadas em dois grandes grupos: as escolhas baseadas em memória e as escolhas baseadas em estímulos (Rottenstreich, Sood, \& Brenner, 2007). As decisões baseadas em memória estão associadas a uma carga cognitiva maior, pois envolvem combinar características e avaliações mentais de uso de diferentes produtos e sustentar estas diferentes opções na memória de trabalho (Rottenstreich et al., 2007). Quando a comparação de dois produtos, por exemplo, a construção das imagens mentais acontece no rascunho visuoespacial e oferece uma visão simulada de como um produto ou serviço pode atender às necessidades dos consumidores (MacInnis \& Price, 1987; Jiang, Gorn, Galli, \& Chattopadhyay, 2016), este processo leva a demandas mais intensas da memória de trabalho. Diferentemente, as escolhas baseadas em estímulos possuem uma orientação puramente emocional, com poucas exigências da memória de trabalho (Rottenstreich et al., 2007).

Apesar da relevância que a memória de trabalho tem na construção das decisões de compra e no comportamento do consumidor, ainda são escassos os estudos disponíveis que procuram explicar os efeitos dos estímulos de marketing na memória de trabalho (Rumpf et al., 2015). Pesquisas nesta área poderão favorecer tanto ao Marketing - elucidando mecanismos que operam informações de propagandas e esforços publicitários -, bem como à área da Saúde Mental, visto que disfunções no comportamento de consumo podem estar relacionadas ao funcionamento da memória de trabalho. Este artigo se propõe a trazer uma revisão narrativa a respeito da memória de trabalho no escopo de comportamento do consumidor e oferecer algumas oportunidades interessantes de estudo para aprofundar o conhecimento a respeito do funcionamento deste construto em uma perspectiva mercadológica.

\section{MÉTODO}

Rother (2007) afirma que a revisão narrativa é mais ampla e possui o propósito de discutir o "estado da arte" de um determinado assunto, e, diferentemente de uma revisão sistemática, apresenta um rigor metodológico menor. Consiste em um método exploratório e a seleção de artigos é feita de forma arbitrária, o que autoriza maior liberdade ao autor se comparada com a análise sistemática (Ferenhof \& Fernandes, 2016). Este tipo de olhar sobre a literatura oferece uma análise qualitativa sobre o conhecimento disponível, sob um ponto de vista contextual. 0 levantamento do "estado da arte" busca compreender a produção científica dentro de um determinado cenário e seu propósito atrai uma análise educacional sobre um determinado tema (Vosgerau \& Romanowski, 2014).

Ainda que este tipo de revisão possa trazer consigo um viés proveniente da não exaustão do tema na busca bibliográfica, é possível, através da revisão narrativa, ter a liberdade de adotar uma perspectiva teórica a respeito do assunto e oferecer um ponto de vista mais harmonioso em termos dos elementos conceituais abordados (Faro \& Pereira, 2013). Apesar desta metodologia não requerer um rigor quantitativo de levantamento dos registros, optamos por oferecer uma visão em termos da frequência obtida na pesquisa dos artigos, para que a carência de publicações sobre o assunto se tornasse mais evidente.

A busca e seleção dos artigos nacionais foi realizada através das palavras-chave "memória de trabalho" e working memory em quatro plataformas que indexam publicações na específicas na área de Administração e Negócios: Redalyc, DOAJ, SPELL e Portal Capes. Os artigos internacionais foram procurados e selecionados na plataforma Ebsco Business Source Complete. Como o volume de artigos tanto em um como em outro caso foi baixo, não se estabeleceu um corte de datas, sendo incluídos todos os artigos encontrados até o fim do ano de 2019. Valentim et al. (2017) utilizaram uma interessante forma visual para ilustrar a construção do corpus de pesquisa bibliográfica, que demonstra os temas abordados, fruto das buscas realizadas, e as palavras chave utilizadas no processo de busca (Figura 1). 


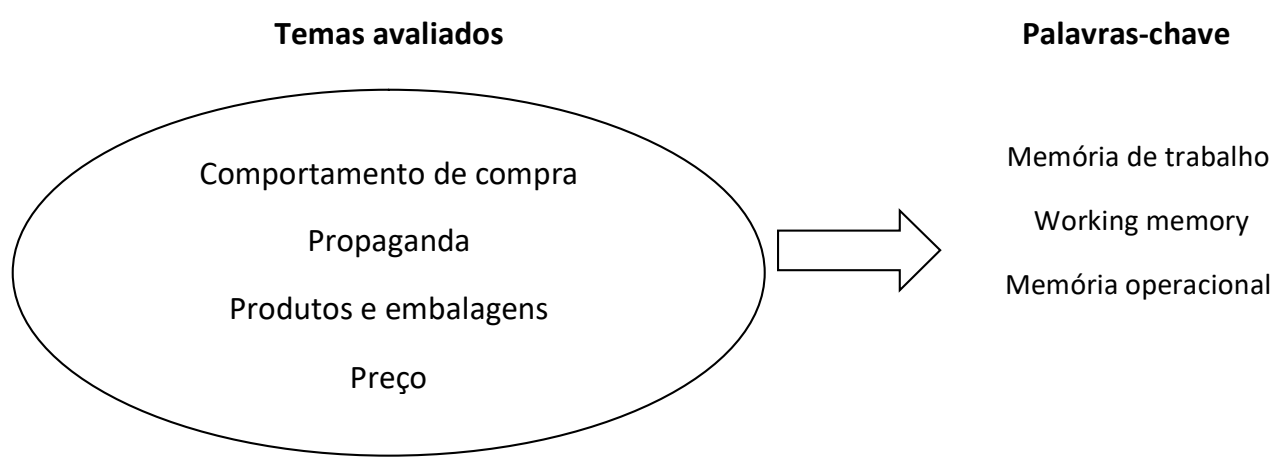

Fonte: Adaptado de Valentim et al. (2017)

Figura 1. Temas principais abordados e as palavras chave utilizadas

\section{RESULTADOS}

A busca nas plataformas Scielo, SPELL e DOAJ não retornou nenhum artigo relacionado, ainda que remotamente, com a área de Marketing. Somente a Redalyc retornou 24 artigos, entre os quais apenas 5 foram selecionados, pois os demais tratavam do tema fora do escopo de Marketing. No portal Capes foram identificados 4 artigos, todos eles já apresentados na busca anterior, que totalizaram 5 artigos como representantes de publicações nacionais. A plataforma Ebsco retornou 740 artigos disponíveis, todos dentro do escopo de negócios, porém apenas 33 elegíveis no contexto mercadológico (os excluídos abordavam a memória de trabalho áreas como gestão de pessoas, percepção de risco, liderança, ente outros). Nem todos estes selecionados possuem escopo experimental, mas trazem informações relevantes sobre o processamento de informações mercadológicas na memória de trabalho.

Tabela 1

Resumo da busca de artigos

\begin{tabular}{lcc}
\multicolumn{1}{c}{ Indexador } & $\begin{array}{c}\text { Publicações sobre o tema em } \\
\text { revista de negócios }\end{array}$ & \begin{tabular}{c} 
Publicações selecionadas \\
\hline Redalyc (artigos nacionais)
\end{tabular} \\
DOAJ (artigos nacionais) & 24 & 5 \\
SPELL (artigos nacionais) & 0 & 0 \\
Scielo (artigos nacionais) & 0 & 0 \\
Portal Capes (artigos & 0 & 4 \\
nacionais) & 4 & 33 \\
Ebsco Business Source & 740 & \\
(artigos internacionais) & & \\
\hline
\end{tabular}

Fonte: Elaborado pelos autores.

\section{DISCUSSÃO}

Ainda que não se tenha feito uma revisão sistemática da literatura, os resultados são suficientes para observar a grande oportunidade de publicações de experimentos que relacionem memória de trabalho e estímulos de marketing. Observado primeiramente o resultado com os artigos nacionais, quatro entre os cinco artigos possuem algo em comum: o construto é representado apenas como apoio teórico na argumentação dos trabalhos e não possuem escopo experimental.

Outra peculiaridade observada é que, nestes mesmos estudos, a memória de trabalho é tratada em um mesmo sentido da memória de curto prazo (Dias, Maffezzolli, \& Pinheiro, 2016; Duarte \& Korelo, 2017; Junior, Damacena, \& Bronzatti, 2015; Silva \& Urdan, 2008), junção que ainda não se pode estabelecer de forma conclusiva. Aben e colaboradores (2012) apresentam seis possíveis relações entre a memória de trabalho e a memória de curto prazo, e em apenas uma delas admite-se serem construções sobrepostas. Sem dúvida são estruturas que apresentam íntima relação, considerando o fato de os estudos demonstrarem uma correlação de 0.7 a 0.9 entre tarefas que envolvem as memórias 
de curto prazo e de trabalho, contudo, há uma porção da variância que é particular a cada construto (Aben, Stapert, \& Blokland, 2012).

Endo e Roque (2017) realizaram um estudo a respeito da atenção visual em uma propaganda de bebida e como elementos da cena são percebidos pelos participantes. Os autores relatam a função armazenadora e integradora da memória de trabalho e sustentam sua importância no processamento da propaganda, porém, desconsideram que esta estrutura multicomponente possui elementos modais e, particularmente, uma de suas principais atribuições é o controle do foco de atenção. Diferente da estrutura passiva descrita, a memória de trabalho teria uma função ativa não só no processamento online das informações sensoriais, mas também, segundo Baddeley e colaboradores (2012), em ponderar emocionalmente estas informações.

Vários estudos internacionais buscam compreender o elo entre propaganda e memória de trabalho. 0 imaginário é fruto de um processo mental no qual a informação sensorial do ambiente e a informação da memória de longo prazo interagem na memória de trabalho e o produto deste processo possui implicações importantes para os estrategistas de propagandas, visto que estas construções no imaginário orientarão a efetividade das suas campanhas (Mccracken \& Macklin, 1998). 0 resultado desta interação poderá levar a diferentes níveis de engajamento dos consumidores com os produtos e a forma como a imagem mental é construída e associada ao produto ou serviço poderá afetar o juízo que o consumidor terá a respeito da marca, ainda sem nunca a ter experimentado (Jiang et al., 2016).

A construção mental de imagens acontece no componente visuoespacial da memória de trabalho - é lá que são realizadas as manipulações cognitivas do conteúdo visual. 0 consumidor poderá projetar-se em uma narrativa de um comercial, imaginando-se utilizar o produto ou estabelecendo algum tipo de relacionamento com a marca e estas construções mentais acontecerão na memória de trabalho. Por exemplo, Bolls e Lang (2003) avaliaram o uso de recursos cognitivos na propaganda de rádio. Diferentemente do que poderia se imaginar, onde apenas seria exigido recursos do componente fonológico, os resultados indicaram uma demanda cognitiva maior porque recrutaria também o rascunho visuoespacial para construção das imagens mentais oferecidas pela comunicação. Isto significa dizer que o imaginário possibilita "ver" pelo rádio graças às informações consolidadas na memória de trabalho. E a forma como interagem o conteúdo verbal e fonológico na memória de trabalho levaria a diferentes resultados na interpretação do conteúdo (Bolls \& Lang, 2003).

0 detector hedônico pode ter um papel fundamental no processamento da comunicação, pois é ele que conduz ao "despertar" (arousal) emocional e sustenta emocionalmente as construções metais (Moore \& Lee, 2013). Imagens induzidas pela propaganda podem levar um viés positivo codificado na memória (Potter et al., 2006) devido à ação do detector hedônico. Um anúncio visto antes do encontro do consumidor com o produto ou serviço pode influenciar a memória de trabalho ao longo da experiência, isto é, leva o consumidor a "ver o que espera ver" - a propaganda pode ajudar ou prejudicar a maneira como a experiência é codificada na memória de longo prazo devido a um rol de expectativas presentes na memória de trabalho (Braun-LaTour \& LaTour, 2005). As crenças e promessas construídas pela publicidade e presentes na memória de longo prazo podem se materializar no imaginário construído neste componente cognitivo.

A limitação da memória de trabalho deveria ser uma preocupação de todo publicitário quando realiza uma propaganda. Há um tempo muito exíguo para transmitir uma determinada quantidade de informações, mas, dependendo da quantidade de dados que são inseridos na memória de trabalho, esta pode se esgotar rapidamente e o efeito da propaganda ser mais prejudicial do que favorável (Haverty \& Blessing, 2007). De outro lado, estudos mostram que a propaganda que ativa os dois componentes modais (visual e verbal) é mais efetiva se comparada à estimulação de somente um deles (Weisstein, Kukar-Kinney, \& Monroe, 2016). Embora a aprendizagem diminua quando a memória de trabalho está sobrecarregada (e.g.: processando textos e informações pictóricas, simultaneamente), a capacidade efetiva pode ser aprimorada quando as informações são apresentadas por meio de múltiplos canais sensoriais (eg.: auditivo, visual e háptico), diferente do que aconteceria se apenas um canal fosse utilizado (Besharat et al., 2013). Isto mostra que os dois componentes modais podem operar de forma aditiva e colaborativa no processo de memorização do consumidor.

Quando uma informação de texto é apresentada juntamente com uma narração, pode ser processada por dois canais sensoriais, tornando o conteúdo com maior probabilidade de memorização 
(Weisstein et al., 2016). Brasel e Gips (2013) encontraram efeito positivo na memória dos consumidores para propagandas que utilizaram legendas no mesmo idioma acompanhando a narrativa do filme, comparada com a mesma propaganda onde havia somente o áudio com o conteúdo narrado. Os autores concluíram que o uso de legendas aprimorou a efetividade da comunicação porque: (a) a apresentação simultânea do texto visual com áudio pode ajudar na compreensão de informações mais complexas, em relação a apenas com o áudio; (b) a integração de informações visuais e verbais podem proporcionar a categorização emocional mais rápida além de reduzir o esforço cognitivo e, por último, (c) ajuda a aprimorar a lembrança para informações e marcas divulgadas no comercial, uma vez que o texto sincronizado com o conteúdo visual proporciona melhor compreensão e reduz a carga cognitiva.

As embalagens são como "vendedores silenciosos" que, por meio da comunicação visual, buscam capturar a atenção do consumidor (McNeal \& Ji, 2003). Contudo, em um típico supermercado, há mais de 40.000 itens à venda, com diversas categorias e versões. 0 consumidor não perde muito tempo no processo de seleção. A sua escolha acontecerá em condições de tempo restrito e sob uma grande quantidade de opções (Reutskaja et al., 2011). Os componentes modais especializados irão processar o conteúdo capturado pelo foco de atenção, gerenciado pelo executivo central, que opera seletivamente em função da relevância emocional que o sistema atribui aos diferentes produtos ofertados. Pouco se sabe sobre como esta sobrecarga de oferta impacta na dinâmica de processamento da memória de trabalho. "Dicas" externas podem disparar um gatilho para a entrada de informações na memória de trabalho. Estas dicas externas podem ser visuais como, por exemplo, os logotipos nas embalagens dos produtos (Mccracken \& Macklin, 1998) ou características de design (formas e cores) que, capturadas pelo executivo central e ponderadas pelo detector hedônico, disparam considerações mentais a respeito do produto. Na medida em que o consumidor encontra outras "dicas", provenientes de outros produtos ou comunicações no ponto de venda, a memória de trabalho torna-se mais carregada de informações, o que leva o consumidor a ter de repetir de forma subvocal determinadas informações caso queira que elas permaneçam ativas (Biswas, Biswas, \& Chatterjee, 2009).

Um exemplo disso acontece quando há comparações de características entre os produtos: no processo de escolha de uma determinada marca no supermercado, as eleitas como possibilidades de compra têm seus atributos registrados na memória e repetidos mentalmente para proceder à comparação e decisão. Este é um processo dinâmico e ainda pouco estudado na área do comportamento do consumidor. Pouco se sabe a respeito de como as os diferentes elementos de uma embalagem (cor, forma, composição, texto, entre outros) operam na memória de trabalho e quais características de embalagem podem ter maior relevância na manutenção da informação, tanto visual como fonológica (Otterbring et al., 2013). 0 foco de atenção do consumidor está associado com a natureza da valência que atribui às marcas: aquelas com associações negativas ou positivas podem ser identificadas mais rapidamente devido às ligações destas com emoções positivas ou negativas, respectivamente (Faizi \& Szücs, 2010). Não somente as representações mentais, mas também os aspectos geométricos de uma marca podem ser afetados pela memória de trabalho. Acredita-se que a forma circular versus angular de um logotipo ativam associações de suavidade e dureza, respectivamente, e estas associações que são mentalmente construídas no imaginário do consumidor podem influenciar nos julgamentos de atributo de um produto (Jiang et al., 2016).

Em um supermercado, ao longo da jornada de compra, há a operacionalização mental do que se vê sobre produtos e ofertas bem como sua localização. Não é raro termos na mente um composto de produtos e sua localização e termos de abandonar estas informações porque outras, com maior relevância, tomaram o seu lugar. Somos seletivos neste processo. 0 consumidor pode ter identidade com mais do que uma marca, mas a probabilidade de optar por uma ou outra marca será maior para aquela que se fizer mais presente na memória de trabalho (Marin, Ruiz, \& Rubio, 2009). A interação do consumidor com uma marca é resultado de um processo cognitivo que reúne um conjunto de percepções e avaliações operacionalizadas na memória de trabalho (Stocchi et al., 2003). Os diferentes significados que uma marca pode representar são formulados na memória de trabalho e essa interpretação possui importantes implicações para o comportamento de compra ( $\mathrm{Ng}$ \& Houston, 2009). Somada ao viés social, a limitação da capacidade de armazenamento na memória de trabalho faz com que os consumidores utilizem diferentes níveis de informações para construção de suas 
percepções das marcas, informações estas oriundas tanto da memória de longo prazo como do ambiente (Rumpf et al., 2015).

Um conceito central no marketing e na gestão de marcas é o brand awareness e refere-se à capacidade de ativação de uma determinada marca na mente do consumidor. A limitação da memória de trabalho leva o brand awareness a ser seletivo e a possibilidade de uma marca ser ativada estaria diretamente relacionada à eficiência e intensidade com que ela consegue se apresentar na memória de trabalho, proveniente da memória de longo prazo (Woodside \& Walser, 2007). Esta eficiência na recuperação estaria associada ao despertar emocional (arousal) que uma marca provoca e é fundamental no processo de construção do Brand Equity (Huang \& Sarigöllü, 2012), que é definido como o sobrevalor que o cliente concorda em pagar para adquirir uma marca específica em detrimento de outras (Woodside \& Walser, 2007). Para uma marca desconhecida, os consumidores têm pouca ou nenhuma informação armazenada na memória e a falta de familiaridade reduz a faixa de preço aceitável a ser paga pelo produto (Weisstein et al., 2016). Parece razoável pensar que a avaliação deste importante indicador de marketing possa ser feita por tarefas cognitivas que permitam mensurar a eficiência de resgate da marca para a memória de trabalho e não apenas através de pesquisas convencionais de mercado. 0 tempo de resposta seria utilizado como uma estimativa da efetividade desta ativação (Pearson et al., 2014). Uma marca conseguirá se distinguir das demais na relação direta do quanto melhor for o desempenho no processo de captura e transferência desta marca da memória de longo prazo para este espaço de decisão (Woodside \& Walser, 2007). Isto faz com que as diferentes marcas venham a competir não somente pelo espaço em gôndola, mas também pelo espaço na memória de trabalho do consumidor.

Uma das estratégias do marketing para afetar as decisões de compra dos consumidores são os descontos de preços (Choi \& Coulter, 2012). A retenção das informações fonológicas relativas aos valores é uma função da memória de trabalho. Para decidir por uma oferta ou outra, o consumidor necessita realizar cálculos mentais que, por vezes, podem sobrecarregar a capacidade de memória de trabalho, e o esforço promocional (no caso de descontos de preços) pode ter um efeito diferente do esperado (Choi \& Coulter, 2012). O trabalho computacional infringido ao consumidor (eg.: calcular desconto sobre desconto) pode gerar desinteresse e afastamento devido à grande carga cognitiva. Os consumidores têm modelado o armazenamento de informações de preços na memória de trabalho de acordo com sua experiência e a importância da informação do preço para a decisão de compra (Gaston-Breton \& Raghubir, 2014).

Durante o processo de comparação de preços entre produtos, há uma disputa por um "espaço" na memória de trabalho, que será ponderada por fatores como o vínculo emocional com a marca e as vantagens associadas ao produto, funções do detector hedônico e do executivo central (Suri et al., 2012). 0 processo de comparação de preços pode ser realizado com diferentes níveis de eficiência face às diferenças individuais na capacidade da memória de trabalho, e o resultado pode orientar diferentes comportamentos de compra. Quando a informação fonológica de preço é codificada de uma maneira mais eficiente, há mais espaço na memória de trabalho para integrar outros elementos no processo de decisão. De outro lado, componentes longos de preços podem sobrecarregar a capacidade da memória de trabalho (e.g.: "quarenta e sete reais e vinte e nove centavos" ocupa um espaço maior do que "quarenta e oito reais") (Luna \& Kim, 2009) e deixar poucos recursos para que o consumidor possa considerar outros elementos no seu processo de decisão.

Os preços com nomenclatura longa podem sobrecarregar o loop fonológico e levar a estimativas menos precisas do preço total de uma cesta de compras. Além disso, fatores relacionados a atenção podem influenciar o processo de fragmentação/cálculo através do componente executivo central da memória de trabalho (Luna \& Kim, 2009). Esta limitação pode mascarar algumas estratégias de práticas de preço (caso o consumidor priorize armazenar outras informações do produto, que não o preço), contudo ainda são poucos os estudos a respeito. Praticamente inexistem trabalhos que explorem a relação entre a capacidade da memória de trabalho, comparação de preços e o processo de decisão.

Há evidências de que os consumidores codificam os preços em três tipos de códigos - verbal, visual e de magnitude - e que os diferentes consumidores têm diferentes tendências ou habilidades para confiar em cada código (Vanhuele, Laurent, \& Drèze, 2006). 0 código verbal está associado à 
evocação correta do preço. Indica a lembrança numérica do valor armazenado. A memorização visual possui uma perspectiva mais pictórica do que fonológica, uma vez que o armazenamento do valor acontece mediante um código visual. A codificação do preço em termos de magnitude está relacionada à ordem de grandeza numérica e dicotômica, discriminada nos critérios de mais caro ou mais barato. Isto acontece quando memória de trabalho é ativada de forma implícita, quando não há conhecimento consciente de informações de preços ou de forma explícita, quando as informações de preços são processadas conscientemente e depois armazenadas na memória (Gaston-Breton \& Raghubir, 2014), muitas vezes repetidas mentalmente (subvocal) para garantir a perpetuação no armazenamento.

\section{CONSIDERAÇÕES FINAIS}

A forma como a memória é tratada em boa parte da literatura de Marketing ainda é limitada em sua estrutura conceitual e sustentada sobre os conceitos da memória de longo prazo, especificamente nos fundamentos das memórias semântica (conhecimento do mundo vigente) e autobiográfica (experiências individuais anteriores) (Ratnayake Broderick, \& Mitchell, 2010). Não há dúvidas de que a informação armazenada na memória de longo prazo é determinante para o processo de decisão e influencia diretamente nas decisões do consumidor (Ratnayake et al., 2010). Contudo, os processos de decisão acontecem on-line, quando o consumidor está diante das opções, decidindo entre os produtos A, B ou C. É neste momento que a memória de trabalho assume um protagonismo ainda pouco estudado.

Do ponto de vista da gestão de marketing, é importante fazer com que os consumidores tenham o menor dispêndio da capacidade de memória de trabalho para o processamento de informações de marcas e produtos. E mais, garantir que a informação seja marcante ao ponto de não ceder o seu lugar a outra. A comunicação de marketing deve, portanto, considerar a capacidade cognitiva de grupos-alvo específicos, bem como a frequência e a duração da comunicação devem ser ajustadas à capacidade do grupo-alvo processar cognitivamente tais informações (Rumpf et al., 2015). Por exemplo, a capacidade da memória de trabalho decresce com a idade, o que levaria a comunicação para idosos ser diferente da usada para os mais novos. Outro ponto seria a dinâmica de distrações, o que levaria à necessidade de os gerentes colocarem informações sobre a marca em situações em que a intensidade do contexto seja bastante baixa (presença de estímulos que possam dispersar a memória de trabalho) para facilitar a atenção visual do grupo-alvo.

Inúmeras são as oportunidades de estudos, em uma perspectiva interdisciplinar entre a Psicologia e o Marketing. Os números de artigos publicados mostram abertura e propensão a esta análise. Muito se tem produzido na Psicologia sobre a memória de trabalho pautado em estudos laboratoriais, com pouca aplicação no cotidiano das pessoas. Há uma base neurocientífica muito rica que permite fundamentar experimentos e estudos de Marketing para compreender de forma mais naturalística os processos cognitivos das pessoas. Estudos futuros, sem dúvida, permitirão compreender melhor como o processo de decisão de compra é construído no ambiente da memória de trabalho, tendo em vista os seus múltiplos componentes. Há oportunidades para estudar a relação entre os componentes da memória de trabalho e, por exemplo, o processamento de comunicação, ou de comparação de preços, assim como seu papel nos estudos ligados a merchandising, comunicação e promoção relacionados ao componente visuoespacial do modelo.

A relevância de direcionar estudos neste sentido se aprofunda na medida em que novas tecnologias vão surgindo. Um grande desafio é compreender como o volume de informação afeta as novas gerações. Atualmente, um ambiente multitelas (TV, tablet e celular) faz com que os indivíduos se tornem "multitarefas": é comum vermos ao nosso redor pessoas curvadas em seus celulares, interagindo em um ambiente virtual, enquanto executam outras atividades. Pouco se sabe, todavia, qual o impacto que esta simultaneidade de tarefas possui na capacidade de processamento e armazenamento da memória de trabalho, e como este comportamento pode comprometer (ou se de fato compromete) o processo de codificação das informações e o desempenho dos indivíduos em suas tarefas cotidianas. Praticamente inexistem publicações a respeito. Esta é uma seara quase inexplorada que sugere, merece e demanda investigação e atenção dos estudiosos de Marketing e Psicologia. 


\section{Referências}

Aben, B., Stapert, S., \& Blokland, A. (2012). About the distinction between working memory and shortterm memory. Frontiers in Psychology, 3(August), 301. http://doi.org/10.3389/fpsyg.2012.00301

Allen, R. J., Baddeley, A., \& Hitch, G. J. (2006). Is the binding of visual features in working memory resource-demanding? Journal of Experimental Psychology: General, 135(2), 298-313. http://doi.org/10.1037/0096-3445.135.2.298

Alloway, T. P., Gerzina, A., \& Moulder, R. (2016). Investigating the Roles of Affective Processes, Trait Impulsivity, and Working Memory in Impulsive Buying Behaviors. Comprehensive Psychology, 5, 1-9. http://doi.org/10.1177/2165222816659640

Argyriou, E., \& Melewar, T. C. (2011). Consumer attitudes revisited: a review of attitude theory in marketing research. International Journal of Management Reviews, 13(4), 431-451. http://doi.org/10.1111/j.1468-2370.2011.00299.x

Baddeley, A. (1996). Exploring the central executive. The Quarterly Journal of Experimental Psychology, $49 A(1), 5-28$.

Baddeley, A. (2002). Is Working Memory Still Working? European Psychologist, 7(2), 85-97. http://doi.org/10.1027//1016-9040.7.2.85

Baddeley, A. (2013). On applying cognitive psychology. British Journal of Psychology, 104(4), 443-56. http://doi.org/10.1111/bjop.12049

Baddeley, A., Banse, R., Huang, Y.-M., \& Page, M. (2012). Working memory and emotion: Detecting the hedonic detector. Journal of Cognitive Psychology, 24(1), 6-16. http://doi.org/10.1080/20445911.2011.613820

Barcelos, R. H., \& Rossi, C. A. V. (2015). A Contribuição da Produção Científica em Marketing para as Ciências Sociais. Revista de Administração Contemporânea, 19(spe2), 197-220. http://doi.org/10.1590/1982-7849rac20151740

Biswas, D., Biswas, A., \& Chatterjee, S. (2009). Making judgments in a two-sequence cue environment: The effects of differential cue strengths, order sequence, and distraction. Journal of Consumer Psychology, 19(1), 88-97. http://doi.org/10.1016/j.jcps.2008.12.011

Besharat, A., Kumar, A., Lax, J. R., \& Rydzik, E. J. (2013). Leveraging Virtual Attribute Experience in Video Games to Improve Brand Recall and Learning. Journal of Advertising, 42(2-3), 170-182. http://doi.org/10.1080/00913367.2013.774593

Bleckley, M. K., Foster, J. L., \& Engle, R. W. (2015). Working memory capacity accounts for the ability to switch between object-based and location-based allocation of visual attention. Memory \& Cognition, 43(3), 379-388. http://doi.org/10.3758/s13421-014-0485-z

Bolls, P. D., \& Lang, A. (2003). I Saw It on the Radio: The Allocation of Attention to High-Imagery Radio Advertisements. Media Psychology, 5(1), 33-55. http://doi.org/10.1207/S1532785XMEP0501_2

Bose, M., Folse, J. A. G., \& Burton, S. (2013). The role of contextual factors in eliciting creativity: primes, cognitive load and expectation of performance feedback. Journal of Consumer Marketing, 30(5), 400-414. http://doi.org/10.1108/JCM-02-2013-0475

Braun-LaTour, K. A., LaTour, M. S., \& Zinkhan, G. M. (2007). Using Childhood Memories to Gain Insight into Brand Meaning. Journal of Marketing. http://doi.org/10.1509/jmkg.71.2.45

Choi, P., \& Coulter, K. S. (2012). It's Not All Relative: The Effects of Mental and Physical Positioning of Comparative Prices on Absolute versus Relative Discount Assessment. Journal of Retailing, 88(4), 512-527. http://doi.org/10.1016/j.jretai.2012.04.001

Corbin, J., McElroy, T., \& Black, C. (2010). Memory reflected in our decisions: Higher working memory capacity predicts greater bias in risky choice. Judgment and Decision Making, 5(2), 110-115.

Dias, A. G. G., Maffezzolli, E. C. F., \& Pinheiro, M. A. (2016). A influência da saliência da música na memória de curto prazo e na atitude à marca e ao anúncio. REMark - Revista Brasileira de Marketing, 15(4), 540-553. http://doi:10.5585/remark.v15i4.3413 
Duarte, N. P., \& Korelo, J. C. (2017). A utilização do protocolo verbal think aloud para rastreamento de processos em pesquisas sobre a tomada de decisão do consumidor. REMark - Revista Brasileira de Marketing, 16(3), 317-333.

Eriksson, J., Vogel, E. K., Lansner, A., Bergström, F., \& Nyberg, L. (2015). Neurocognitive Architecture of Working Memory. Neuron, 88(1), 33-46. http://doi.org/10.1016/j.neuron.2015.09.020

Ester, E. F., Ho, T. C., Brown, S. D., \& Serences, J. T. (2014). Variability in visual working memory ability limits the efficiency of perceptual decision making. Journal of Vision, 14, 1-12. http://doi.org/10.1167/14.4.2.doi

Evans, J. S. B. T. (2003). In two minds: Dual-process accounts of reasoning. Trends in Cognitive Sciences. http://doi.org/10.1016/j.tics.2003.08.012

Faro, A., \& Pereira, M. E. (2013). Medidas do estresse: uma revisão narrativa. Psicologia, Saúde \& Doenças, 14(1), 101-124. http://doi.org/10.1590/S0004-282X2003000500014

Faizi, A. Z., \& Szücs, M. D. (2010). The Cognitive Neuroscientific Foundations of Brand Memory Unravelling Non-declarative Brand Memory. International Business. Copenhagen Business School (CBS).

Retrieved

from http://studenttheses.cbs.dk/xmlui/bitstream/handle/10417/1226/ahmad_zaki_faizi_og_michel l_daniel_szucs.pdf?sequence $=1$

Ferenhof, H. A., \& Fernandes, R. F. (2016). Desmistificando a revisão de literatura como base para redação científica: método SSF. Revista ACB, 21(3), 550-563. http://doi.org/10.20873/uft.24474266.2017v3n3p327

Hammou, A. K., Galib, M. H., \& Melloul, J. (2013). The Contributions of Neuromarketing in Marketing Research. Journal of Management Research, 5(4), 20. https://doi.org/10.5296/jmr.v5i4.4023

Haverty, L. A., \& Blessing, S. B. (2007). What Did That \$2.5 Million Ad Buy Us? Cognitive Science Goes to the Super Bowl. In Proceedings of the Annual Meeting of the Cognitive Science Society (347352).

Huang, R., \& Sarigöllü, E. (2012). How brand awareness relates to market outcome, brand equity, and the marketing mix. Journal of Business Research, 65(1), 92-99. http://doi.org/10.1016/j.jbusres.2011.02.003

Janiszewski, C., Kuo, A., \& Tavassoli, N. T. (2013). The Influence of Selective Attention and Inattention to Products on Subsequent Choice. Journal of Consumer Research, 39(6), 1258-1274. http://doi.org/10.1086/668234

Jiang, Y., Gorn, G. J., Galli, M., \& Chattopadhyay, A. (2016). Does your company have the right logo? How and why circular- and angular-logo shapes influence brand attribute judgments. Journal of Consumer Research, 42(5), 709-726. http://doi.org/10.1093/jcr/ucv049

Junior, J. C. S. P., Damacena, C., \& Bronzatti, R. (2015). Pré-ativação: o efeito priming nos estudos sobre o comportamento do consumidor. Estudos e Pesquisas em Psicologia, 15(1), 284-309.

Kahneman, D. (2011). Thinking, fast and slow. New York: Farrar, Straus and Giroux.

Lazer, W., \& Kelley, E. J. (1960). Interdisciplinary Horizons in Marketing. Journal of Marketing, 25(2), 24. http://doi.org/10.2307/1248607

Lobo, F. S., Acrani, I. O., \& Ávila, C. R. B. De. (2008). Tipo de estímulo e memória de trabalho fonológica. Revista CEFAC, 10(4), 461-470. http://doi.org/10.1590/S1516-18462008000400006

Luna, D., \& Kim, H. C. (2009). How much was your shopping basket? Working memory processes in total basket price estimation. Journal of Consumer Psychology, 19, 346-355. http://doi.org/10.1016/j.jcps.2009.03.003

Marin, L., Ruiz, S., \& Rubio, A. (2009). The Role of Identity Salience in the Effects of Corporate Social Responsibility on Consumer Behavior. Journal of Business Ethics, 84(1), 65-78. http://doi.org/10.1007/s10551-008-9673-8

Mccracken, J. C., \& Macklin, M. C. (1998). The role of brand names and visual cues in enhancing memory for consumer packaged goods. Marketing Letters, 9(2), 209-226.

McNeal, J. U., \& Ji, M. F. (2003). Children's visual memory of packaging. Journal of Consumer Marketing, 20(5), 400-427. http://doi.org/10.1108/07363760310489652 
Moore, D. J., \& Lee, S. P. (2013). How advertising influences consumption impulses- The Role of Visualization, Anticipated Emotions, Taste Anticipation, and Hedonic Rationalization. Journal of Abnormal Psychology, 41(3), 107-120. http://doi.org/10.2753/JOA0091-3367410307

Murray, J.D., Jaramillo, J., \& Wang, X.J. Working memory and decision-making in a frontoparietal circuit model. Journal of Neuroscience. 2017;37(50):12167-12186. doi:10.1523/JNEUROSCI.034317.2017

Ng, S., \& Houston, M. J. (2009). Field dependency and brand cognitive structures. Journal of Marketing Research, XLVI(April), 279-292.

Otterbring, T., Shams, P., Wa, E., \& Gustafsson, A. (2013). Left isn't always right: placement of pictorial and textual package elements. British Food Journal, 115(8), 1211-1225. http://doi.org/10.1108/BFJ-08-2011-0208

Pearson, B., Rǎ, J., Bays, P. M., Pertzov, Y., \& Husain, M. (2014). Working memory retrieval as a decision process. Journal of Vision, 14(2), 1-15. http://doi.org/10.1167/14.2.2

Potter, R. F., LaTour, M. S., Braun-LaTour, K. A., \& Reichert, T. (2006). The Impact of Program Context on Motivational System Activation and Subsequent Effects on Processing a Fear Appeal. Journal of Advertising, 35(3), 67-80. http://doi.org/10.2753/J0A0091-3367350305

Radvansky, G., \& Copeland, D. (2006). Memory retrieval and interference: Working memory issues. Journal of Memory and Language, 55(1), 33-46. http://doi.org/10.1016/j.jml.2006.02.001

Ratnayake, N., Broderick, A. J., \& Mitchell, R. L. C. (2010). A neurocognitive approach to brand memory. Journal of Marketing Management, 26(13-14), 1295-1318. http://doi.org/10.1080/0267257X.2010.522531

Repovs, G., \& Baddeley, A. (2006). The multi-component model of working memory: explorations in experimental cognitive psychology. Neuroscience, 139(1), 5-21. http://doi.org/10.1016/j.neuroscience.2005.12.061

Reutskaja, E., Nagel, R., Camerer, C. F., \& Rangel, A. (2011). Search Dynamics in Consumer Choice under Time Pressure: An Eye-Tracking Study. American Economic Review, 101(2), 900-926. http://doi.org/10.1257/aer.101.2.900

Rother, E. T. (2007). Revisão sistemática X Revisão da Literatura. Acta Paulista de Enfermagem, 20(2), v-vi.

Rumpf, C., Noël, B., Breuer, C., \& Memmert, D. (2015). The Role of Context Intensity and Working Memory Capacity in the Consumer's Processing of Brand Information in Entertainment Media. Psychology \& Marketing, 32(7), 764-770. http://doi.org/10.1002/mar.20816

Silva, M. A., \& Urdan, A. T. (2008). O conhecimento do consumidor sobre preços: um exame de produtos de oito categorias de bens duráveis. Revista de Administração Mackenzie, 9(2), 83-103.

Solnais, C., Andreu-Perez, J., Sánchez-Fernández, J., \& Andréu-Abela, J. (2013, June). The contribution of neuroscience to consumer research: A conceptual framework and empirical review. Journal of Economic Psychology. https://doi.org/10.1016/j.joep.2013.02.011

Stocchi, L., Wright, M., \& Driesener, C. (2003). On the stochastic nature of memory: insights from neuropsychology relevant to brand equity. In Australian \& New Zealand Marketing Academy (pp. 1-7).

Suri, R., Cai, J. Z., Monroe, K. B., \& Thakor, M. V. (2012). Retailers' Merchandise Organization and Price Perceptions. Journal of Retailing, 88(1), 168-179. http://doi.org/10.1016/j.jretai.2011.02.005

Valentim, P. P., Falcão, R. P. de Q., \& Campos, R. D. (2017). O corpo nos estudos de consumo: uma revisão bibliográfica sobre o tema. Consumer Behavior Review, 1(Special Edition), 32-48.

Vanhuele, M., Laurent, G., \& Drèze, X. (2006). Consumers' Immediate Memory for Prices. Journal of Consumer Research, 33(2), 163-172. http://doi.org/10.1086/506297

Vosgerau, D. S. R., \& Romanowski, J. P. (2014). Estudos de revisão: implicações conceituais e $\begin{array}{lllll}\text { metodológicas. } & \text { Revista } & \text { Diálogo }\end{array}$ http://doi.org/10.7213/dialogo.educ.14.041.DS08

Weisstein, F. L., Kukar-Kinney, M., \& Monroe, K. B. (2016). Determinants of consumers' response to pay-what-you-want pricing strategy on the Internet. Journal of Business Research, 69(10), 43134320. http://doi.org/10.1016/j.jbusres.2016.04.005 
Wingfield, A. (2016). Evolution of Models of Working Memory and Cognitive Resources. Ear \& Hearing, $1,35-43$.

Woodside, A. G., \& Walser, M. G. (2007). Building strong brands in retailing. Journal of Business Research, 60(1), 1-10. http://doi.org/10.1016/j.jbusres.2006.09.009 